\title{
Hard X-Ray Focusing with Curved Reflective Multilayers
}

\author{
Christian Morawe ${ }^{1}$ and Markus Osterhoff ${ }^{1,2}$ \\ ${ }^{1}$ X-Ray Optics Group, European Synchrotron Radiation Facility, 6 rue Jules Horowitz, 38043 Grenoble, France \\ ${ }^{2}$ Institute for X-Ray Physics, University of Göttingen, Friedrich-Hund-Platz 1, 37077 Göttingen, Germany
}

Correspondence should be addressed to Christian Morawe, morawe@esrf.fr

Received 24 March 2010; Accepted 21 July 2010

Academic Editor: Ali Khounsary

Copyright ( 92010 C. Morawe and M. Osterhoff. This is an open access article distributed under the Creative Commons Attribution License, which permits unrestricted use, distribution, and reproduction in any medium, provided the original work is properly cited.

This paper provides a comprehensive overview on the utilization of curved graded multilayer coatings as focusing elements for hard X-rays. It concentrates on the Kirkpatrick-Baez (KB) focusing setup that has been developed at 3rd generation synchrotron sources worldwide. The optical performance of these devices is evaluated applying analytical and numerical approaches. The essential role of the multilayer coating and its meridional d-spacing gradient are discussed as well as important technological issues. Experimental data and examples of operational $\mathrm{KB}$ focusing devices and applications complement the work.

\section{Introduction}

Over the last decade, hard X-ray optics has evolved rapidly, accelerated by the intense use of extremely brilliant 3rd generation synchrotron radiation sources. The use of smaller samples, the study of local areas, or the need to scan an object with high spatial resolution pushed the development of focusing devises. At present, X-ray beams can be focused down to spots below $50 \mathrm{~nm}$ using various optical elements such as Fresnel Zone Plates (FZPs) [1], Compound Refractive Lenses (CRLs) [2], Wave Guides (WGs) [3], capillary optics [4], curved Total Reflection Mirrors (TRMs) [5, 6], transmission Multilayer Laue Lenses (MLLs) [7, 8], and Reflective Multilayers (RMLs) $[9,10]$. Several theoretical studies, mainly numerical wave optical calculations, were published treating the cases of FZPs [11], CRLs [12], TRMs $[13,14]$, and MLLs $[15,16]$. Advanced numerical ray tracing calculations have been applied to RMLs $[17,18]$. Alternatively, a purely analytical approach was proposed [19, $20]$. The topic of this publication is the application of curved graded RMLs to KB focusing devices [21]. The KB geometry consists of two perpendicularly oriented curved mirrors and is particularly useful on synchrotron beamlines since it can transform the elongated synchrotron source into a nearly circular spot (Figure 1). Being an off-axis system the device produces a clean spot with low background and does not require beam stops or protecting apertures. A single focusing mirror per dimension operating under grazing incidence, as outlined in chapter 2, is not suitable for imaging applications since the Abbé sine condition [22] is not fulfilled. However, two successive mirrors (elliptic/hyperbolic), the so called Wolter geometry [23], can overcome this limitation.

\section{Basic Optics Considerations}

2.1. Resolution Limits. As in visible light optics the diffraction limited resolution (Full Width at Half Maximum, FWHM) of an X-ray optical element with a flat rectangular aperture is given by

$$
D_{\text {diff }}=\frac{0.44 \cdot \lambda}{\mathrm{NA}},
$$

where NA $=n \cdot \sin \varepsilon$ is the numerical aperture with the opening angle $2 \varepsilon$ and the optical index $n$ and $\lambda$ the wavelength of the radiation. The minimum achievable spot size, however, is also limited by the demagnified image of the source of size $S$

$$
D_{\text {source }}=\frac{q}{p} \cdot S,
$$

where $p$ is the source distance and $q$ the image distance with respect to the optics. The phenomenon can also be treated in 


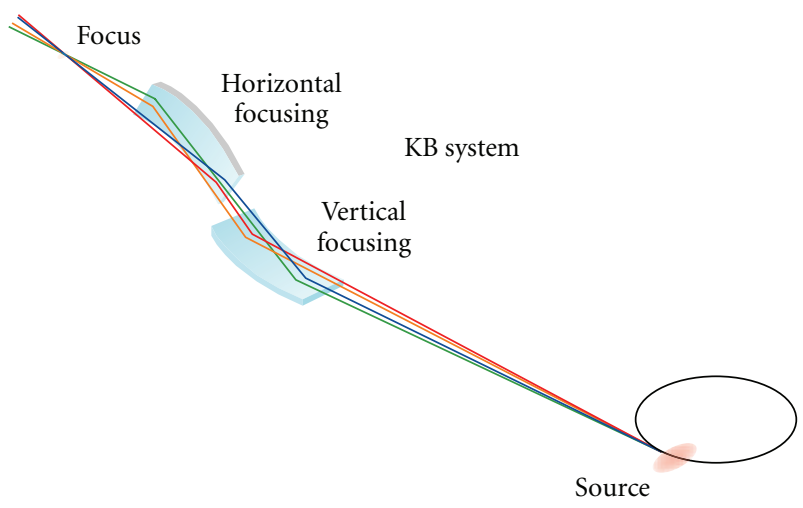

FIGURE 1: Schematic view of a KB focusing setup for a synchrotron source. By proper choice of the respective image distances the elongated source can be focused to a circular spot.

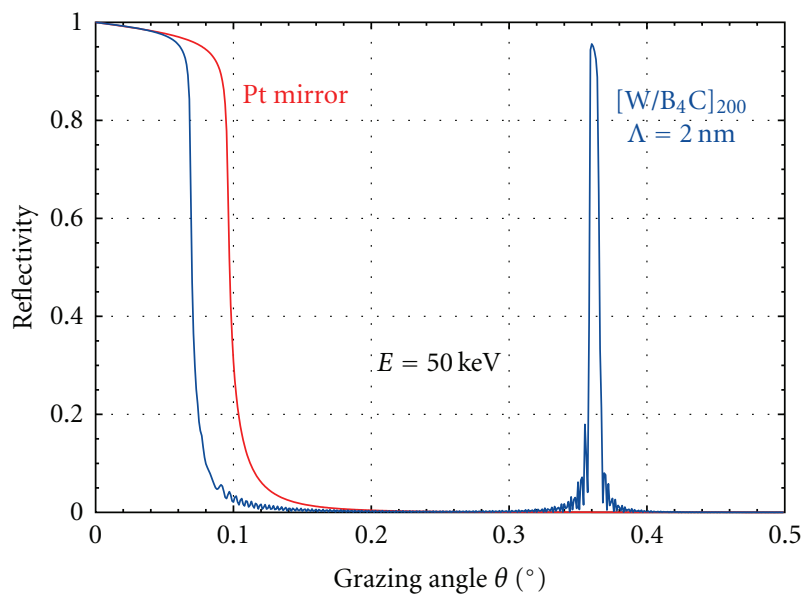

FIgURE 2: Comparison of the simulated reflectivity of a Pt TRM and a W/B $\mathrm{B}_{4} \mathrm{C}$ RML with a d-spacing of $2.0 \mathrm{~nm}$ at a photon energy of $50 \mathrm{keV}$.

terms of the coherence properties of the beam. With a sourcesize limited transversal coherence length [24]

$$
L_{T} \approx \frac{\lambda}{2} \cdot \frac{p}{S}
$$

and assuming that the optical aperture selects only the coherent section of the incoming beam, the diffraction limited spot size becomes

$$
D_{\text {diff }}\left(L_{T}\right) \approx 1.76 \cdot \frac{q}{p} \cdot S \approx D_{\text {source }} .
$$

This means that, apart from a geometrical factor, the diffraction limited spot size of a coherently illuminated optical element is of the order of the size of the source image. Additional performance limiting effects of real optics are figure errors, scattering from defects or nonuniform areas, and further experimental obstacles like alignment, vibration, or drift.

Whilst the short wavelength of hard X-rays alone would principally allow for a spot size far below the nm scale, the main drawback in X-ray optics remains the low achievable numerical aperture. The latter is caused by the weak interaction of high-energy photons with matter, as given by the complex optical index

$$
n=1-\delta+i \beta \approx 1
$$

where $\delta$ describes the dispersion and $\beta$ the absorption in the material. Most development efforts in focusing optics for hard X-rays are therefore dedicated to increasing the numerical aperture.

In the case of reflecting optical elements and neglecting phase shifts upon reflection, the ideal geometry for a pointto-point focusing setup would be an ellipsoid. Since this work deals with $\mathrm{KB}$ setups operated at very small grazing angles, the scheme can be very well approximated by perpendicular projections on two elliptic cylinders. Thus, the treatment can be reduced to two individual ellipses.

2.2. Total Reflection Mirrors. In the case of a single elliptic TRM the range of grazing angles is limited to the upper side by the critical angle of total external reflection $\theta_{C}$ (Figure 2) given by

$$
\sin \theta_{C}=\sqrt{2 \cdot \delta}
$$

and to the lower side only by the physical mirror length when approaching the minimum angle of incidence at the intersection point with the semiminor axis of the ellipse. Since $\delta$ is a small quantity for hard X-rays, the achievable opening $2 \varepsilon$ is very limited. A quantitative estimate can be made assuming a full opening of up to half of the critical angle [9].

$$
4 \cdot \varepsilon \leq \theta_{C}
$$

leading to a diffraction limit of

$$
D_{\text {diff }}(\mathrm{TRM}) \approx \frac{1.76 \cdot \lambda}{\sqrt{2 \cdot \delta}}=1.76 \sqrt{\frac{\pi}{r_{0} \cdot \rho_{e}}},
$$

where $r_{0}$ is the classical electron radius and $\rho_{e}$ the electron density of the material. This means that the focusing power of a TRM is entirely limited by material properties. For a $\mathrm{Pt}$ mirror one obtains a minimum spot size of about $25 \mathrm{~nm}$ FWHM. It should be noted that, in contrast to other techniques, TRM focusing has the advantage of being nondispersive.

2.3. Reflective Multilayers. Considerable improvement can be obtained by the use of RMLs where a stack of periodic bilayers reflects the incoming wave field. Provided the ML dspacing $\Lambda$ matches the modified Bragg equation

$$
\Lambda=\frac{\lambda}{2 \sqrt{n^{2}-\cos ^{2} \theta}},
$$

at any point along the mirror, constructive interference enhances the reflectivity up to values that can reach $90 \%$ or more. The Bragg angle of a RML can be several times 
bigger than the critical angle of a TRM. Figure 2 shows a comparison of the simulated reflectivity between a Pt TRM and a 200-period W/B $\mathrm{B}_{4} \mathrm{C}$ RML at a photon energy of $50 \mathrm{keV}$.

The typical number of bilayers in RMLs is of the order of 100, leading to an energy resolution in the percent range. In conjunction with high peak reflectivity, this means that RMLs can provide an integrated reflectivity exceeding those of perfect crystals by two orders of magnitude. Flat RMLs are well understood and have been used as X-ray optical elements for many years $[25,26]$. Thanks to the flexible d-spacing and the choice of suited material pairs the optical performance of RMLs can be optimized according to photon energy, incidence angle, and further experimental parameters. This choice is also affected by properties like interface diffusion, stress, and chemical stability.

Instead of periodic RMLs, nonperiodic layered structures can be deposited to obtain broader reflectivity profiles [27]. In combination with meridional thickness gradients they can also be applied to KB setups [28].

To focus X-rays an RML has to be curved in accordance with the angular dependence

$$
\sin \theta=\frac{b}{\sqrt{p \cdot q}}
$$

of the ellipse where $b$ is its semiminor axis.

As for TRMs, the achievable resolution of curved RMLs can be estimated analytically [9]. Applying the Bragg law without refraction correction

$$
\sin \theta \approx \frac{\lambda}{2 \cdot \Lambda},
$$

which is a good approximation for small d-spacings and low wavelengths, respectively, the diffraction limit can be calculated as

$$
D_{\text {diff }}(\mathrm{RML}) \approx \frac{0.88}{1 / \Lambda_{2}-1 / \Lambda_{1}}
$$

Here, $\Lambda_{1,2}$ indicate the ML d-spacings at the respective edges of the mirror. The resolution is therefore limited only by the lateral d-spacing gradient of the RML. For a short period RML with strong gradient $(\Lambda=2, \ldots, 3 \mathrm{~nm})$ focal spots of about $5 \mathrm{~nm}$ FWHM appear realistic. Equation (12) can be compared with the corresponding formula for a linear Fresnel Zone Plate (FZP)

$$
D_{\text {diff }}(\mathrm{FZP})=0.88 \cdot \Delta R
$$

with outermost zone width $\Delta R$. Considering a RML as an equivalent to half of a linear zone plate $(1 / 2 \mathrm{FZP})$ with outermost repetition period $\Lambda_{2}=2 \cdot \Delta R$ and setting the largest ML d-spacing $\Lambda_{1}$ to infinity (half of central FZP zone) one obtains

$$
D_{\text {diff }}\left(\frac{1}{2} \mathrm{FZP}\right)=0.88 \cdot \Lambda_{2}=D_{\text {diff }}(\mathrm{RML})
$$

Equation (14) shows that, within the given approximation, both optical elements are different representations of the same focusing configuration (Figure 3 ).

For applications it is interesting to note that none of the above equations (8) or (12) contains any explicit energy dependence.

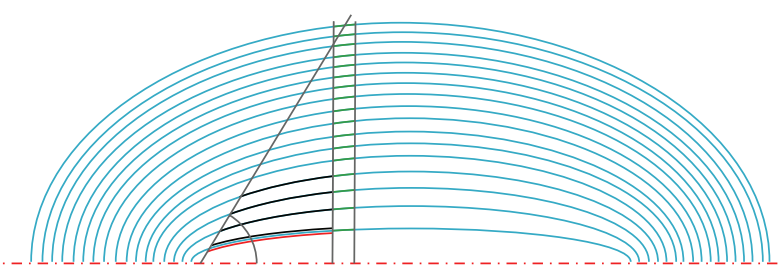

RML TRM FZP

FIGURE 3: TRM, RML, and FZP in the same focusing configuration.

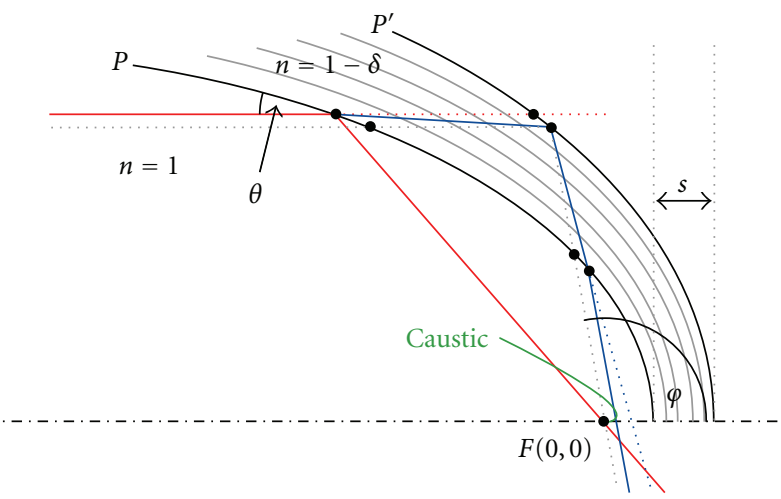

FIGURE 4: Ray propagation through a curved layered structure. Rays reflected from the upper RML surface $P$ reach the ideal focus $F$. Rays reflected at $P^{\prime}$ are refracted when crossing $P$.

\section{Models and Simulations}

3.1. Ray Tracing Calculations. So far the RML was treated as a single reflecting surface. In order to investigate the impact of the beam penetration into the ML structure, both numerical and analytical ray tracing simulations were developed [1820]. An analytical approach is shown in Figure 4 for the case of a parabolic RML. It can be shown that the elliptic case is virtually identical for the high ellipse eccentricities that occur in synchrotron applications. The RML stack is approximated by a single thick layer of average optical index $n=1-\delta$. Multiple reflections and refractions within the stack are neglected, and refraction at the surface $P$ is treated in linear approximation. The aim is to calculate the principal intersection points of rays that penetrate into the structure and that are reflected by the second interface $P^{\prime}$. An ensemble of parallel incoming beams forms an envelope curve of enhanced intensity (caustic) near the ideal focus $F$.

The analytical derivation is carried out to first order in $\delta$, which is a small quantity for hard X-rays. The resulting intersection points of the outgoing beams with the optical axis $X$ on the one hand and with the focal plane $Y$ on the other can be written as

$$
\begin{gathered}
X(y=0) \approx \delta \cdot s \cdot t^{4}, \\
Y(x=0) \approx 2 \cdot \delta \cdot s \cdot t^{3}
\end{gathered}
$$

with

$$
t=\tan \frac{\varphi}{2}
$$


TABLE 1: Comparison of the transmitted energy bandwidth of different optical elements.

\begin{tabular}{cccc}
\hline & FZP & CRL & RML \\
\hline$d E / E$ & $D^{2} /(1.76 \cdot f \cdot \lambda)$ & $D^{2} /(3.52 \cdot f \cdot \lambda)$ & $D^{2} /(3.52 \cdot \Delta f \cdot \lambda)$ \\
\hline
\end{tabular}

and $s$ being as indicated in Figure 4. Rays impinging at lower grazing angles $\theta$ produce stronger aberrations in both $x$ and $y$ directions. Both the layer thickness and the refracting power of the medium amplify the effect. The situation is illustrated in Figure 5 for a source distance $p=150 \mathrm{~m}$, an image distance $q=0.077 \mathrm{~m}$, and a photon energy $E=24550 \mathrm{eV}$.

Figure 6 shows the intersection points $Y$ with the focal plane given in Figure 5 as a function of the grazing angle $\theta$. The purple line represents a calculation using the approximation (15) while the red line was obtained by exact numerical ray tracing on the same structure. Both curves are in good agreement for higher angles. Closer to the critical angle $\theta_{C}$, where the linear approximation of Snell's law fails, the curves diverge. The solid circles are independent calculations made at Osaka University using a different ray tracing code. There is a reasonably good agreement between the two methods [29]. The examples in Figures 5 and 6 were calculated for a parameter set corresponding to realistic focusing setups at a 3rd generation synchrotron beamline. The expected aberrations in the focal plane would be lower than $10 \mathrm{~nm}$ for a typical grazing angle of $0.3^{\circ}$.

The analytical approach has the benefit that equations (15) allow for an estimate of chromatic aberrations and for a comparison with other types of focusing elements. Chromaticity enters through the energy dependent refractive decrement

$$
\delta \propto \frac{1}{E^{2}} .
$$

It can be shown that [20], for a given optical element, the tolerated bandwidth $d E / E$ for a given focal spot size $D$ (FWHM) takes the form summarized in Table 1. It has to be underlined that, in contrast to FZPs and CRLs, where the macroscopic focal length $f$ appears in the denominator, only the small aberration $\Delta f \cong X$ enters the formula for the RML, which makes this element nearly achromatic. The physical background of the different impact on the chromaticity is clearly visible. For FZPs and CRLs the phase shift induced by the optical elements is at the origin of their focusing properties, and chromaticity is therefore unavoidable. In the case of RMLs, however, it is rather a parasitic effect that should be suppressed as much as possible.

It has to be emphasized that the above analysis is based on ideal elliptically or parabolically shaped RMLs. It is known, however, that the optimum shapes of focusing RMLs differ from these perfect mathematical curves. It is a general practice to assume that RMLs have to fulfill Bragg's law (9) locally $[30,31]$ in order to provide constructive interference at the same photon energy and at any point along the optical surface. The ML d-spacings and consequently the interface positions in space change as the optical indices differ from

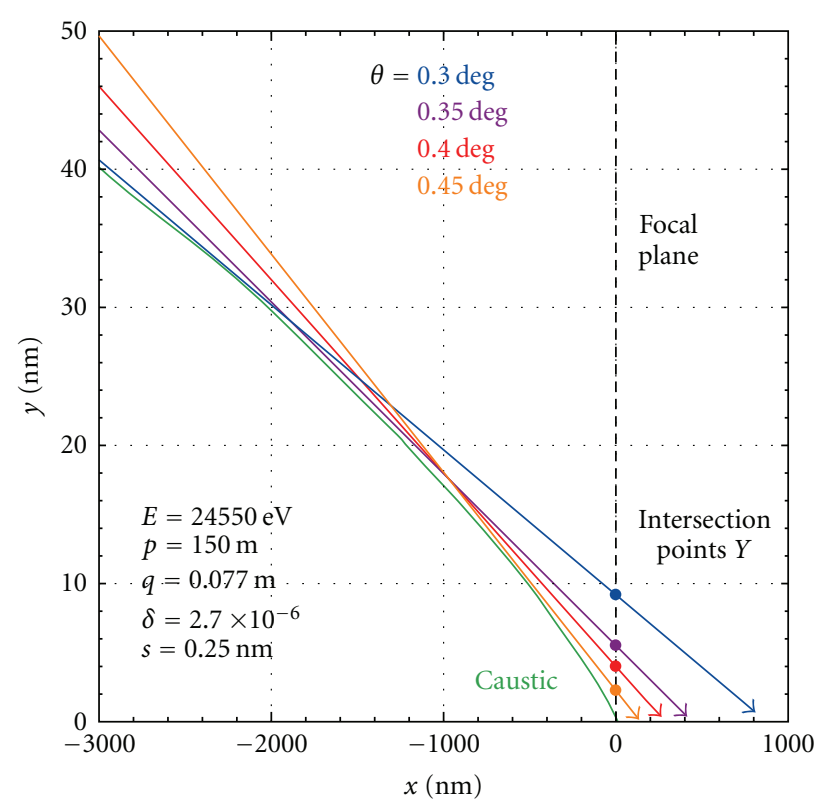

FIGURE 5: Ensemble of rays (straight arrows) emerging from a RML under variable grazing angles and constant $s$. The lower thick curve indicates the corresponding caustic.

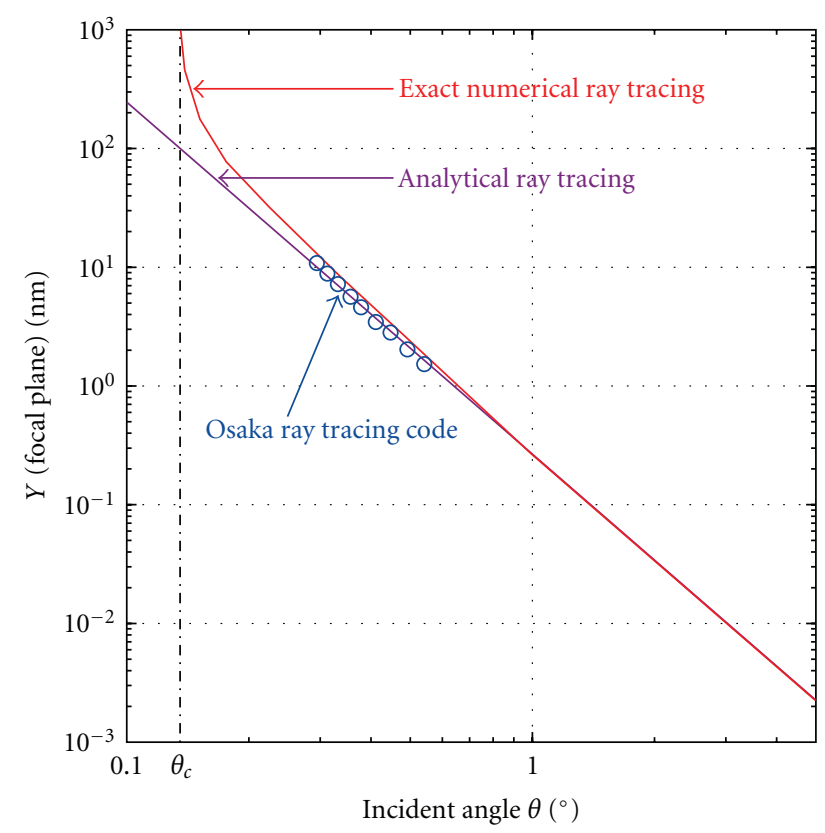

FIGURE 6: Intersection points of the rays with the focal plane as a function of the grazing angle. The purple line was derived from (15), the red curve shows exact ray tracing calculations. Blue circles indicate simulated data from Osaka University.

unity. There is strong evidence that this refraction correction implicitly leads to the optimum RML shape [18]. Full wave optical computations will be required to derive structures that minimize or suppress aberrations. They will also have to include effects caused by figure errors and by the finite source size. 


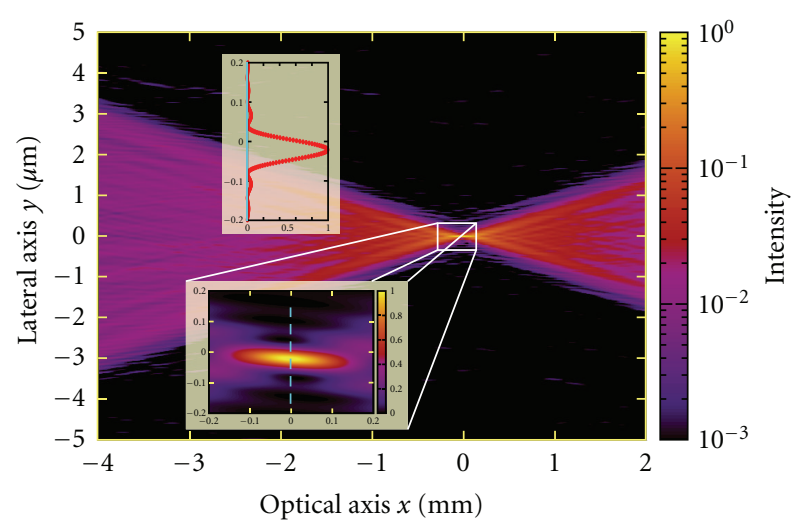

FIGURE 7: Field intensity of a point source focused by an ideal elliptic Pd mirror (logarithmic scale).

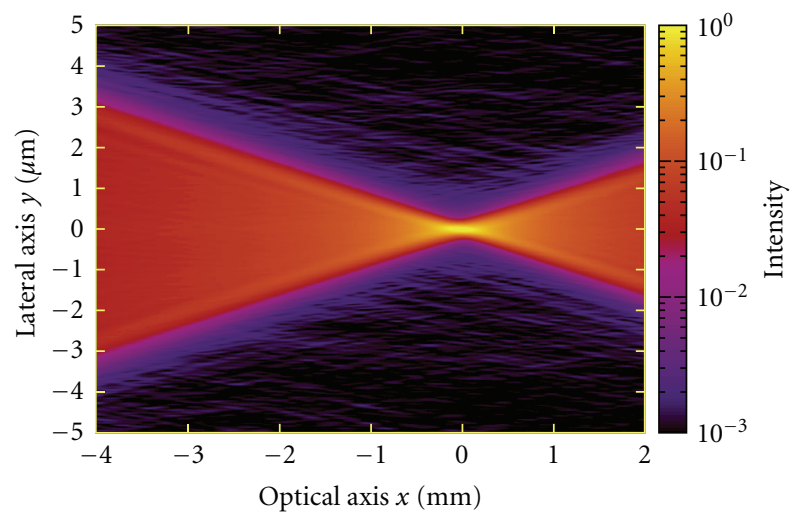

Figure 8: Partially coherent superposition of the focused fields of different point sources representing a spatially extended source (logarithmic scale). Figure errors on the Pd mirror are included.

3.2. Wave Optical Calculations. A first step to minimize the aberration of RMLs is wave optical calculations for a single reflecting surface and the related wave propagation in free space from the source to the mirror and further to the focal plane. An isotropic and monochromatic point source emits a number of rays (typically ten thousands), which are traced onto the mirror's surface $S$, where they are reflected. The amplitude is multiplied by the corresponding Fresnel coefficient, so the outgoing field $A(s)$ is known in amplitude and phase. In a region around the nominal focus point the intensity distribution is calculated by a numerical solution of Kirchhoff's integral of diffraction, treated here in two dimensions corresponding to the plane of incidence $(x, y)$ :

$$
I(x, y)=\left|\frac{1}{\sqrt{\lambda}} \int_{S} A(s) \cdot \frac{e^{i k r}}{\sqrt{r}} \cdot d s\right|^{2} .
$$

Figure 7 shows the field intensity emitted by an isotropic point source and focused by an ideal elliptic Pd mirror at a photon energy of $8 \mathrm{keV}$. Source and image distances are $p=$ $85 \mathrm{~m}$ and $q=0.2 \mathrm{~m}$, respectively. The angle of incidence at the mirror center was set to $4 \mathrm{mrad}=0.23^{\circ}$.

The geometrical cone of the converging beam, the diffraction waist, as well as interference features from the

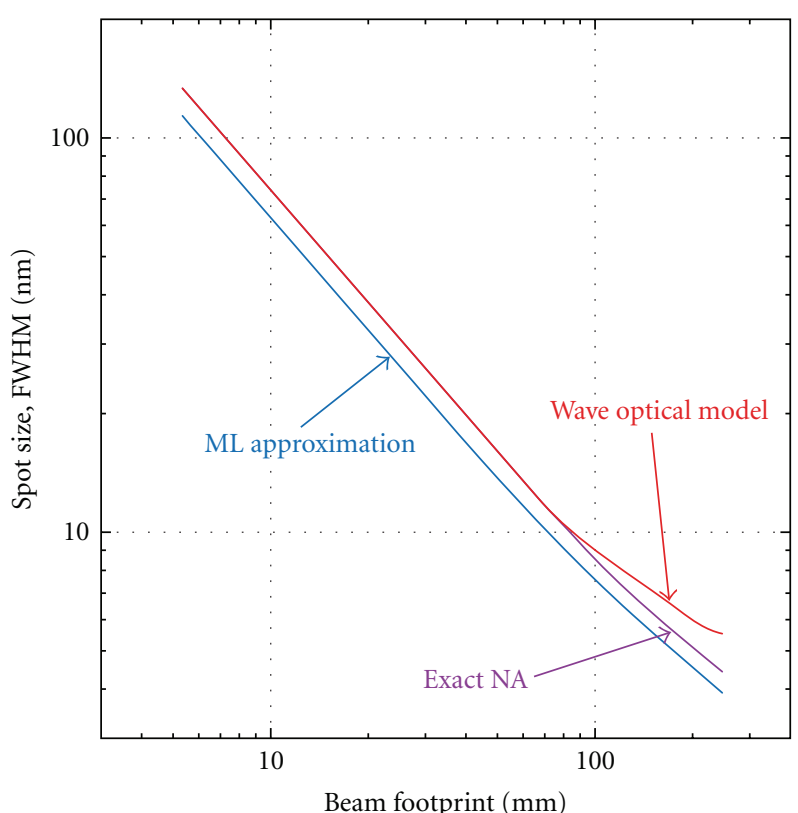

FIGURE 9: Diffraction limited focal spot size (FWHM) versus beam footprint on an elliptic RML, calculated from equation (12) (blue curve), from the exact NA (purple curve), and using a wave optical code for a single surface (red curve).

finite aperture are clearly visible. In the present case, a diffraction limited spot size of $71 \mathrm{~nm}$ FWHM was obtained. The model was refined to treat extended sources as well as figure errors on the mirror surface. Figure 8 shows the performance of the same Pd mirror as in Figure 7, this time assuming a Gaussian source of $85 \mu \mathrm{m}$ FWHM and a figure error of about $5 \mathrm{~nm}$ peak-to-valley (PV). The simulation was made for an ensemble of partially coherent point sources representing a spatially extended source. The focal spot size has increased to $190 \mathrm{~nm}$. The blurring and the disappearance of the interference fringes are mainly due to the finite and only partially coherent source.

It is of interest to compare wave optical results with those that can be obtained by purely geometrical considerations as outlined in Section 2. Figure 9 shows the diffraction limited focal spot size versus the illuminated footprint along a mirror, calculated from (12) (blue curve), from the exact NA (purple curve), and using a wave optical code (red curve). The parameters used in the calculations correspond to those given in Figure 5. The incoming beam aperture is increased while remaining centered at $\theta=0.35^{\circ}$.

It can be seen that the geometrical approach assuming a flat numerical aperture NA (thin lens approximation) agrees well with the wave optical calculations for short mirror sections. As the length of the footprint becomes comparable with the image distance, the geometrical approach underestimates the spot size. In the latter case, the strongly asymmetric aperture can no longer be approximated by a thin lens. Equation (12) returns systematically lower values than the other methods, mainly due to the neglected refraction correction. Despite the observed differences, all 
TABLE 2: Comparison of the experimental performance of various X-ray optical elements.

\begin{tabular}{|c|c|c|c|c|c|c|}
\hline Type & & Reflecting & & & & Refracting \\
\hline Element & TRM & RML & WG & FZP & MLL & CRL \\
\hline$D[\mathrm{~nm}]$ & 25 & 7 & $<50$ & 15 & 16 & $<50$ \\
\hline$E[\mathrm{keV}]$ & $<20$ & $<60$ & $<20$ & $<10$ & $<20$ & $<100$ \\
\hline$\Delta E / E$ & - & $1 \%-10 \%$ & - & $0.1 \%$ & $0.01 \%-0.1 \%$ & - \\
\hline Geometry & Deflecting KB & Deflecting KB & In-line KB & In-line $\mathrm{KB}$ & In-line $\mathrm{KB}$ & In-line $\mathrm{KB}$ \\
\hline Preference & Focusing & Focusing & Holography & Imaging & Imaging & Imaging \\
\hline
\end{tabular}

three descriptions return values that are consistent within the given degree of simplification.

\section{Technological Options}

4.1. Overview. At present, three alternative approaches have been developed to produce curved RMLs for applications at 3rd generation synchrotron sources. APS (USA) investigated profile coatings to provide optical surfaces with the required fixed focus elliptic curvature [6], essentially without ML coatings. Work at the ESRF (France) concentrated on ML coated flat mirrors integrated into dynamical benders to approach the optimum curvature [32]. SPring- 8 and the University of Osaka (Japan) developed deterministic polishing techniques [33] and differential deposition to obtain ultra precise surfaces and the required ML coatings [34]. $\mathrm{KB}$ focusing systems for X-rays, though mainly based on TRMs, are commercially available from various suppliers. A nonexhaustive list is given in [35-41].

4.2. ML Deposition. The principal challenge in the fabrication of an RML is the accurate deposition of the dspacing gradient that fulfills (9). In order to guarantee both uniform reflectivity and phase the tolerated thickness errors of the individual layers must be far below the width of the ML Bragg peak. In practice, the accuracy must be of the order of $0.5 \%$ or better. Today, the most common deposition method is magnetron sputtering. It combines high deposition rates $(0.1-1.0 \mathrm{~nm} / \mathrm{s})$ with reasonably stable operation over a full coating cycle. The high kinetic energy of sputtered particles favors the formation of smooth and dense thin layers. Relative motion between sputter source and substrate, sometimes complemented by the insertion of masks, is the method of choice to produce the correct thickness gradient. This technique is also known as differential deposition.

4.3. Alternative Solutions. An interesting alternative focusing scheme was proposed by Montel [42] where the two perpendicular reflecting surfaces are positioned side by side. This approach offers a more compact arrangement and enables equal demagnification in both directions. The critical issue is the corner line where both mirrors are in contact. Single reflection optics with ellipsoidal shape is attractive thanks to its higher transmission and potentially larger collection angle. The fabrication is particularly challenging in terms of both figuring and polishing. Both types of optics are commercially available [43-46] and are mainly used in combination with laboratory X-ray sources.

\section{Experimental Progress}

With Pt coated mirrors diffraction limited spot sizes of about $25 \mathrm{~nm}$ were measured [47]. More recently, this value could be significantly improved by the use of Pt/C RMLs. During experiments at Spring-8, a line focus of $7 \mathrm{~nm}$ could be obtained with a monochromatic beam at $20 \mathrm{keV} \mathrm{[10].}$ This remarkable performance was achieved by inserting an online wave front correction mirror to account for residual figure errors on the focusing element itself [48]. An example of a line profile is given in Figure 10, containing both experimental data and a numerical simulation (courtesy $\mathrm{H}$. Mimura). It is important to note that these experimental findings are in line with the theoretical estimates made in Sections 2 and 3.

The main drawback of fixed focus KBs is their lack of flexibility required for operation at variable energies. Dynamical setups based on bent RMLs, that offer the possibility to switch the photon energy, have achieved best results of about $45 \mathrm{~nm}$ [49]. So far, figure errors and mechanical vibrations were identified as principal obstacles to reach smaller spots.

\section{Comparison with Other Focusing Elements}

It is not within the scope of this paper to provide a full comparison of the various optical elements presently used to focus hard X-rays. Nevertheless, Table 2 gives a brief overview on the typical experimental performance along some key parameters such as the smallest achieved spot size $D$, typical energy range $E$, intrinsic bandwidth $\Delta E / E$, and practical aspects concerning geometry and preferred mode of operation. It is not exhaustive and subject to ongoing evolution. The corresponding references and abbreviations were introduced in Section 1. A similar summary was published in [50]. A comprehensive overview on the different types of X-ray optics can be found in [51].

\section{Applications}

Over the last 10 years, at ESRF, more than 20 RML based $\mathrm{KB}$ focusing devices were delivered to different beamlines. 


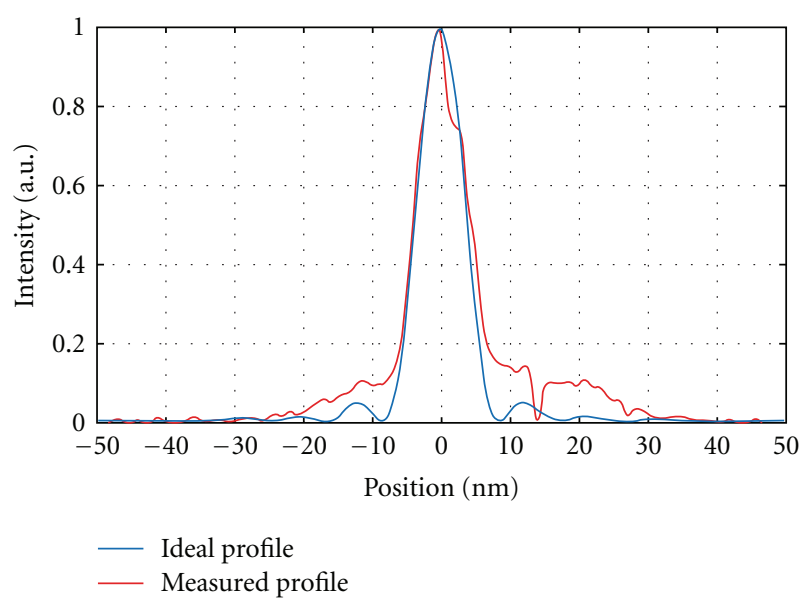

FIGURE 10: Experimental intensity profile (red curve) through the focal line of a curved RML fabricated at Osaka University and measured at Spring- 8 . The blue curve shows the ideal shape of the diffraction limited case (courtesy H. Mimura).

They provide focal spots from $100 \mu \mathrm{m}$ down to less than $100 \mathrm{~nm}$ to the user community. In contrast to crystal optics the high reflectivity and the broad bandwidth of the ML elements allow for high intensity, making them the system of choice for flux limited experiments. The most brilliant ESRF $\mathrm{KB}$ nanofocusing setups provide flux densities of about 3 . $10^{5} \mathrm{ph} / \mathrm{s} / \mathrm{mA} / \mathrm{nm}^{2}$. Novel techniques like projection tomography [52] and fluorescence imaging [53] on the nanometer scale have evolved thanks to the excellent performance of this optical system. They provide new opportunities for various scientific and engineering disciplines.

\section{Summary and Outlook}

KB focusing devices based on graded RML coatings play a major role in present day X-ray optics, in particular on 3rd generation synchrotron beamlines. Thanks to their ML coating their numerical aperture can be increased by a factor of about 5 compared with TRMs. They are widely used and have generated diffraction limited focal spots down to $7 \mathrm{~nm}$ FWHM. While both design and fabrication of curved RMLs have developed rapidly over the last decade, a full wave optical description is still lacking.

Several approaches to further reduce the spot size might be considered. The use of higher-order Bragg peaks would increase the numerical aperture, on the expense of reflectivity and flux. A real technological breakthrough would be the use of two coherently illuminated curved RMLs facing each other. A rough estimate shows [9] that such a setup would reduce the diffraction limit by a factor 4 compared to a single element. If one adds a second RML on each side, leading to two successive reflections (Wolter geometry), the gain in aperture would be about 8 .

From a fundamental point of view the $1 \mathrm{~nm}$ limit seems to be within reach. The task to align several optical elements to within atomic distances, however, represents a major technological challenge.

\section{Acknowledgments}

The authors would like to thank the members of the Department of Precision Science and Technology, Graduate School of Engineering, Osaka University (Japan) for their kind support. The research leading to these results has received funding from the European Community's Seventh Framework Programme (FP7/2007-2013) under grant agreement no. 226716.

\section{References}

[1] W. Chao, B. D. Harteneck, J. A. Liddle, E. H. Anderson, and D. T. Attwood, "Soft X-ray microscopy at a spatial resolution better than 15 nm," Nature, vol. 435, no. 7046, pp. 1210-1213, 2005.

[2] C. G. Schroer, O. Kurapova, J. Patommel et al., "Hard X-ray nanoprobe based on refractive x-ray lenses," Applied Physics Letters, vol. 87, no. 12, Article ID 124103, pp. 1-3, 2005.

[3] A. Jarre, C. Fuhse, C. Ollinger, J. Seeger, R. Tucoulou, and T. Salditt, "Two-dimensional hard x-ray beam compression by combined focusing and waveguide optics," Physical Review Letters, vol. 94, no. 7, Article ID 074801, 2005.

[4] D. H. Bilderback, S. A. Hoffman, and D. J. Thiel, "Nanometer spatial resolution achieved in hard $\mathrm{x}$-ray imaging and Laue diffraction experiments," Science, vol. 263, no. 5144, pp. 201203, 1994.

[5] H. Mimura, S. Matsuyama, H. Yumoto et al., "Hard Xray diffraction-limited nanofocusing with Kirkpatrick-Baez mirrors," Japanese Journal of Applied Physics, vol. 44, no. 1619, pp. L539-L542, 2005.

[6] W. Liu, G. E. Ice, J. Z. Tischler et al., "Short focal length Kirkpatrick-Baez mirrors for a hard x-ray nanoprobe," Review of Scientific Instruments, vol. 76, no. 11, Article ID 113701, pp. 1-6, 2005.

[7] H. C. Kang, J. Maser, G. B. Stephenson et al., "Nanometer linear focusing of hard X rays by a multilayer Laue lens," Physical Review Letters, vol. 96, no. 12, Article ID 127401, pp. $1-4,2006$.

[8] H. C. Kang, H. Yan, R. P. Winarski et al., "Focusing of hard X-rays to 16 nanometers with a multilayer Laue lens," Applied Physics Letters, vol. 92, no. 22, Article ID 221114, 2008.

[9] Ch. Morawe, O. Hignette, P. Cloetens et al., "Graded multilayers for focusing hard X rays below 50nm," in Advances in X-Ray/EUV Optics, Components, and Applications, vol. 6317 of Proceedings of SPIE, 63170F.

[10] H. Mimura, S. Handa, T. Kimura et al., "Breaking the $10 \mathrm{~nm}$ barrier in hard-X-ray focusing," Nature Physics, vol. 6, pp. 122-125, 2009.

[11] F. Pfeiffer, C. David, J. F. van der Veen, and C. Bergemann, "Nanometer focusing properties of Fresnel zone plates described by dynamical diffraction theory," Physical Review B, vol. 73, no. 24, Article ID 245331, 2006.

[12] C. G. Schroer and B. Lengeler, "Focusing hard X rays to nanometer dimensions by adiabatically focusing lenses," Physical Review Letters, vol. 94, no. 5, Article ID 054802, 2005.

[13] K. Yamauchi, K. Yamamura, H. Mimura et al., "Nearly diffraction-limited line focusing of a hard-X-ray beam with an elliptically figured mirror," Journal of Synchrotron Radiation, vol. 9, no. 5, pp. 313-316, 2002.

[14] C. M. Kewish, L. Assoufid, A. T. Macrander, and J. Qian, "Wave-optical simulation of hard-x-ray nanofocusing by precisely figured elliptical mirrors," Applied Optics, vol. 46, no. 11, pp. 2010-2021, 2007. 
[15] C. G. Schroer, "Focusing hard X rays to nanometer dimensions using Fresnel zone plates," Physical Review B, vol. 74, no. 3, Article ID 033405, 2006.

[16] H. Yan, J. Maser, A. Macrander et al., "Takagi-Taupin description of X-ray dynamical diffraction from diffractive optics with large numerical aperture," Physical Review B, vol. 76, no. 11, Article ID 115438, 2007.

[17] H. Mimura, S. Matsuyama, H. Yumoto et al., "Reflective optics for sub-10 nm hard X-ray focusing," in Advances in XRay/EUV Optics and Components II, vol. 6705 of Proceedings of SPIE, 67050L.

[18] H. Mimura, S. Handa, Ch. Morawe et al., "Ray-tracing analysis in aberration of a laterally- graded multilayer mirror," Nuclear Instruments and Methods in Physics Research A, vol. 616, no. 2-3, pp. 251-254, 2010.

[19] J.-P. Guigay, Ch. Morawe, V. Mocella, and C. Ferrero, "An analytical approach to estimating aberrations in curved multilayer optics for hard x-rays: 1 . Derivation of caustic shapes," Optics Express, vol. 16, no. 16, pp. 12050-12059, 2008.

[20] Ch. Morawe, J.-P. Guigay, V. Mocella, and C. Ferrero, "An analytical approach to estimating aberrations in curved multilayer optics for hard $\mathrm{x}$-rays: 2 . Interpretation and application to focusing experiments," Optics Express, vol. 16, no. 20, pp. 16138-16150, 2008.

[21] P. Kirkpatrick and A.V. Baez, "Formation of optical images by X-rays," Journal of the Optical Society of America, vol. 38, p. 766, 1984.

[22] E. Abbe, "über die Bedingungen des Aplanatismus der Linsensysteme," Jenaisch. Ges. Med. Naturw., vol. 129, 1879.

[23] H. Wolter, "Spiegelsysteme streifenden Einfalls als abbildende Optiken für Röntgenstrahlen," Annals of Physics, vol. 445, p. 94, 1952.

[24] J.-A. Nielsen and D. McMorrow, Elements of Modern X-Ray Physics, John Wiley \& Sons, London, UK, 2001.

[25] J. Underwood and T. W. Barbee Jr., "Layered synthetic microstructures as Bragg diffractors for X rays and extreme ultraviolet: theory and predicted performance," Applied Optics, vol. 20, no. 17, pp. 3027-3034, 1981.

[26] Ch. Morawe, "Graded multilayers for synchrotron optics," in Proceedings of the 9th International Conference on Synchrotron Radiation Instrumentation, vol. 879, pp. 764-769, January 2007.

[27] Ch. Morawe, E. Ziegler, J.-C. Peffen, and I. V. Kozhevnikov, "Design and fabrication of depth-graded X-ray multilayers," Nuclear Instruments and Methods in Physics Research A, vol. 493, no. 3, pp. 189-198, 2002.

[28] Ch. Morawe, C. Borel, E. Ziegler, and J.-C. Peffen, "Application of double gradient multilayers for focusing," in X-Ray Sources and Optics, vol. 5537 of Proceedings of SPIE, pp. 115-126, November 2004.

[29] Ch. Morawe, J.-P. Guigay, V. Mocella et al., "Aberrations in curved x-ray multilayers," in Advances in X-Ray/EUV Optics and Components III, vol. 7077 of Proceedings of SPIE, September 2008, 70770T.

[30] M. Schuster and H. Göbel, "Parallel beam coupling into channel-cut monochromators using curved graded multilayers," Journal of Physics D: Applied Physics, vol. 28, p. A270, 1995.

[31] Ch. Morawe, P. Pecci, J.-C. Peffen, and E. Ziegler, "Design and performance of graded multilayers as focusing elements for Xray optics," Review of Scientific Instruments, vol. 70, no. 8, pp. 3227-3232, 1999.
[32] O. Hignette, P. Cloetens, G. Rostaing, P. Bernard, and Ch. Morawe, "Efficient sub $100 \mathrm{~nm}$ focusing of hard X rays," Review of Scientific Instruments, vol. 76, no. 6, pp. 1-5, 2005.

[33] Y. Mori, K. Yamauchi, K. Yamamura et al., "Development of plasma chemical vaporization machining and elastic emission machining systems for coherent X-ray optics," in X-Ray Mirrors, Crystals, and Multilayers, vol. 4501 of Proceedings of SPIE, pp. 30-42, July 2001.

[34] H. Mimura, S. Matsuyama, H. Yumoto et al., "Reflective optics for sub-10 nm hard X-ray focusing," in Advances in XRay/EUV Optics and Components II, vol. 6705 of Proceedings of SPIE, August 2007, 67050L.

[35] http://www.crystal-scientific.com/?KBfocus=.

[36] http://www.idtnet.co.uk.

[37] http://www.irelec.fr/en/produit.asp?idproduit=39.

[38] http://www.j-tec.co.jp/english/focusing/index.html.

[39] http://www.seso.com/uk/index.htm.

[40] http://www.zeiss.de/en.

[41] http://www.winlightx.com.

[42] M. Montel, "X-ray microscopy with catamegonic roof mirrors," in X-Ray Microscopy and Microradiography, pp. 177-185, Academic Pres, New York, NY, USA, 1957.

[43] http://www.axo-dresden.de/indexl.html.

[44] http://www.incoatec.de.

[45] http://www.rigaku.com/optics.

[46] http://www.xenocs.com/accueil.htm.

[47] H. Mimura, H. Yumoto, S. Matsuyama et al., "Efficient focusing of hard x rays to $25 \mathrm{~nm}$ by a total reflection mirror," Applied Physics Letters, vol. 90, no. 5, Article ID 051903, 2007.

[48] T. Kimura, S. Handa, H. Mimura et al., "Wavefront control system for phase compensation in hard X-ray optics," Japanese Journal of Applied Physics, vol. 48, no. 7, Article ID 072503, 2009.

[49] O. Hignette, P. Cloetens, Ch. Morawe et al., "Nanofocusing at ESRF using graded multilayer mirrors," in Proceedings of the 9th International Conference on Synchrotron Radiation Instrumentation (SRI '07), vol. 879, pp. 792-795, January 2007.

[50] A. Snigirev and I. Snigireva, "Hard X-ray microoptics," in Modern Developments in X-Ray and Neutron Optics, A. Erko, M. Idir, T. Krist, and A. G. Michette, Eds., pp. 255-285, Springer, Berlin, Germany, 2008.

[51] http://www.x-ray-optics.com.

[52] R. Mokso, P. Cloetens, E. Maire, W. Ludwig, and J.-Y. Buffire, "Nanoscale zoom tomography with hard x rays using Kirkpatrick-Baez optics," Applied Physics Letters, vol. 90, no. 14, Article ID 144104, 2007.

[53] P. Bleuet, A. Simionovici, L. Lemelle et al., "Hard X-rays nanoscale fluorescence imaging of Earth and Planetary science samples," Applied Physics Letters, vol. 92, no. 21, Article ID 213111, 2008. 